\title{
MONOFRACTAL AND MULTIFRACTAL ANALYSIS IN SHORT - TERM TIME DYNAMICS OF ULF GEOMAGNETIC FIELD MEASURED IN CRETE, GREECE
}

\author{
F. Vallianatos, J. P. Makris, V. Saltas \\ Technological Educational Institute of Crete, Chania, Crete, Greece, Fvallian@chania.teicrete.gr, \\ Jpmakris@chania.teicrete.gr,vsaltas@chania.teicrete.gr \\ L. Telesca, V. Lapenna \\ Istituto di Metodologie per l'Analisi Ambientale, Consiglio Nazionale delle Ricerche, Tito, Italy, \\ Itelesca@imaa.cnr.it
}

\begin{abstract}
In this work, a monofractal and multifractal characterization of the short-term time dynamical fluctuations of the ultra low frequency (ULF) geomagnetic field, measured by one station installed in Creete, Greece, has been carried out. Time scale properties of the three ULF geomagnetic components, two horizontal $(x, y)$ and one vertical $(z)$ have been analyzed through the power spectral density, Higuchi method and Hurst R/S analysis. Results point out the presence of fractal features expressing long-range time correlation with scaling coefficients, which are the clue of persistent mechanism. Using a set of multifractal parameters, defined from the shape of the multifractal spectrum, it has been observed that the degree of multifractality, that characterizes the original signals, is "weaker" if compared to the residual signals, obtained from the original ones after removing the four observed periodicities (24-, 12-, 8- and 6-h periodicties). Furthermore the horizontal $\mathrm{x}$ and $\mathrm{y}$ components have revealed to be less multifractal than the vertical $z$-component.
\end{abstract}

\section{INTRODUCTION}

The investigation of the temporal fluctuations of ultra low frequency (ULF) geomagnetic signals has recently revealed its potential in giving information related to seismotectonic processes. ULF emissions in the frequency range $0.01-10 \mathrm{~Hz}$ were observed using magnetic sensors in very active seismic areas, such as before and after the great crustal earthquakes at Armenia, Spitak on Dec. 7 , 1988 and Loma Prieta, California on Nov. 18, 1989 (Kopytenko et al., 1993; Molchanov et al., 1992). In the study of seemingly complex phenomena such as those generating electromagnetic signals, fractal analysis techniques, developed to draw qualitative and quantitative information from time series, have been applied recently to the study of a large of variety of irregular, erratic signals and by now have proved to be very useful to detect deep dynamical features. These methods are mainly useful to detect the presence of long-range power-law correlations that have been found to exist in a wide variety of systems. As soon as they are found, they are generally quantified by a scaling exponent. By estimating the scaling coefficient we are able to obtain quantitative information on the strength of power-law fluctuations of the signal and to get insight into the kind of mechanisms which may be responsible of its generation. Hayakawa et al. proposed a fractal analysis of ULF data obtained at Guam observatory during a strong $(M s=8.0)$ earthquake occurred on August 8, 1993 near the Guam island (Hayakawa et al., 1999). They found that the spectrum of emissions displayed decreasing power-law behaviour, typical of self-organised critical dynamics, with the scaling exponent gradually decreasing during the process of earthquake preparation. In a later paper, Hayakawa et al. showed that the February 17, 1996, Biak, Indonesia, earthquake was preceded by a decrease of the spectral power-law exponent approaching unity (Hayakawa et al., 2000). Other electromagnetic anomalies were observed in Greece (Tzanis et al., 2000).

The above analyses concern with the determination of a single exponent, leading to a monofractal description of the temporal fluctuations of such kind of signals. Monofractals are 
homogeneous in the sense that they have the same scaling properties, characterized by a single singularity exponent (Stanley et al., 1999). The need for more than one scaling exponent to describe the scaling properties of the process uniquely, indicates that the process is not a monofractal but could be a multifractal. A multifractal object requires many indices to characterize its scaling properties. Multifractals can be decomposed into many -possibly infinitely many- subsets characterized by different scaling exponents. Thus multifractals are intrinsically more complex and inhomogeneous than monofractals. Maybe the most adequate manner to investigate multifractals is to analyze their fractality or singularity spectra. The singularity spectrum quantifies the fractal dimension of the sub-set characterized by a particular exponent that is gives information about the relative dominance of various fractal exponents present in the process. In particular, the maximum of the spectra furnishes the dominant fractal exponent and the width of the spectrum denotes the range of the fractal exponents.

Therefore, the aim of the present work is to perform a multifractal characterization of the time dynamics of three series of ULF geomagnetic data (two horizontal and one vertical components), measured in Crete, Greece, one of the most seismically active areas of the Mediterranean.

\section{GEOLOGICAL AND SEISMOLOGICAL SETTINGS}

The measuring station is located in Crete island, Greece, in the southern Aegean area, one of the most seismically active of the world and one of the most rapidly deforming continental regions. The southern Aegean area is limited to the north by the continental blocks of the European plate, to the south by oceanic material of the African plate, to the east to central Turkey and to the west by the Adriatic sea (Baker et al., 1997). In this region, the African plate is subducted under the Eurasian lithosphere along the Hellenic arc; in this region, the seismic activity is very intense and extends up to a depth of $180 \mathrm{~km}$ (Papazachos, 1990).

The geomagnetic station is equipped with LEMI-009 magnetometer, which has a digital structure and with a full measuring range of $\pm 100,000 \mathrm{nT}$. Furthermore, features of the type of magnetometer used are: very low noise, high temporal and thermal stability and low power consumption (Korepanov et al., 2001). The 1-Hz-sampled geomagnetic field has been continuously measured for two months, April and May 2002. We applied the fractal analysis on the minute means, and the number of samples is sufficient to obtain reliable measures of the multifractal properties.

\section{DATA ANALYSIS}

\subsection{Monofractal analysis}

Before performing the fluctuation analysis of the geomagnetic series, we removed linear trends and then we "studentized" the signals, in order to deal with data having zero mean and unity variance. Fig. 1 shows the "studentized" signals. We firstly performed the power spectral analysis method, which allows detecting significant periodic components of the signals. For long-range correlated signals, that is characterized by a scaling behavior, the power spectral density behaves as a power-law of the frequency $f, P(f) \approx f^{\alpha}$. The results are shown in Fig. 2. Four frequency lines with a relative high amplitude are clearly recognizable. They correspond to the 24-, 12-, 8- and 6-h periodicities. The first two are clearly associated with diurnal and semidiurnal processes (temperature and atmospheric pressure) while the other two frequencies could be higher harmonics of the first ones. The periodic components are superimposed on a scaling behavior, typical of colored-noise processes. 

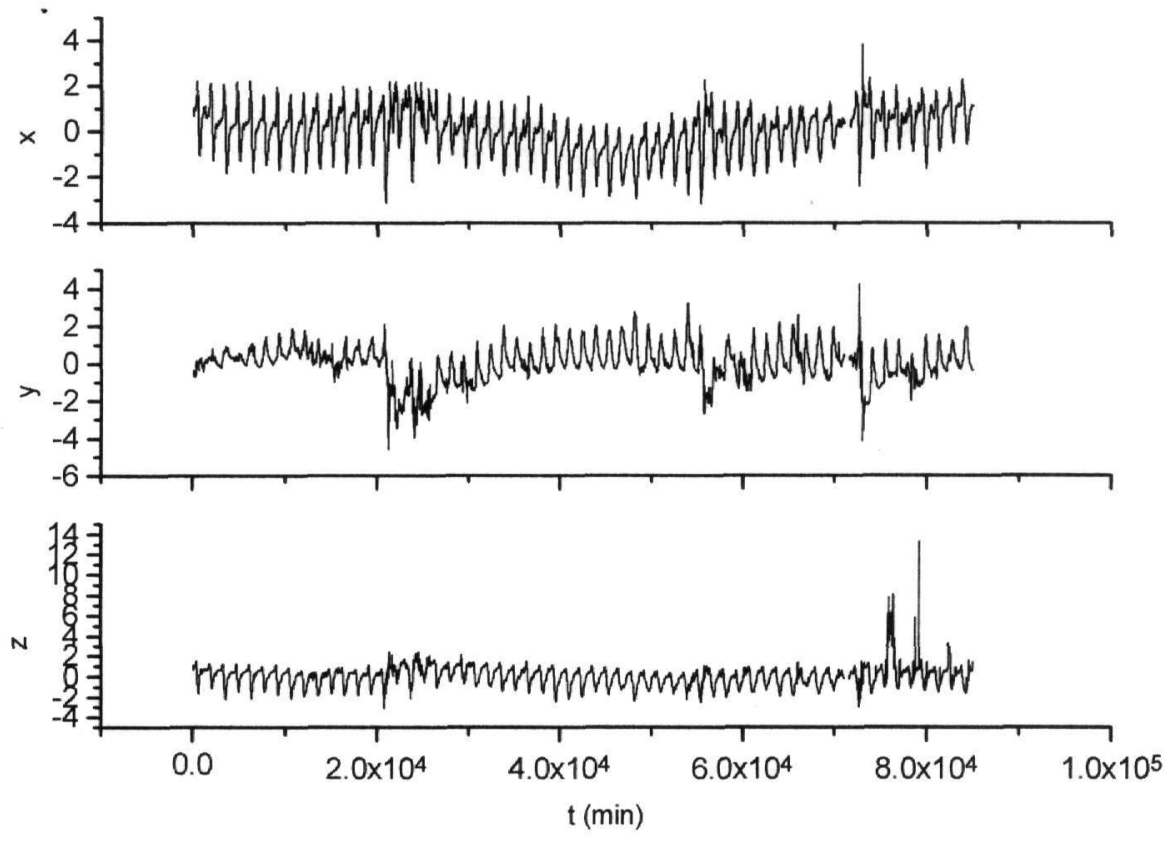

Figure 1. ULF geomagnetic "studentized" signals: horizontal components ( $x$ and $y)$ and vertical component $(z)$.
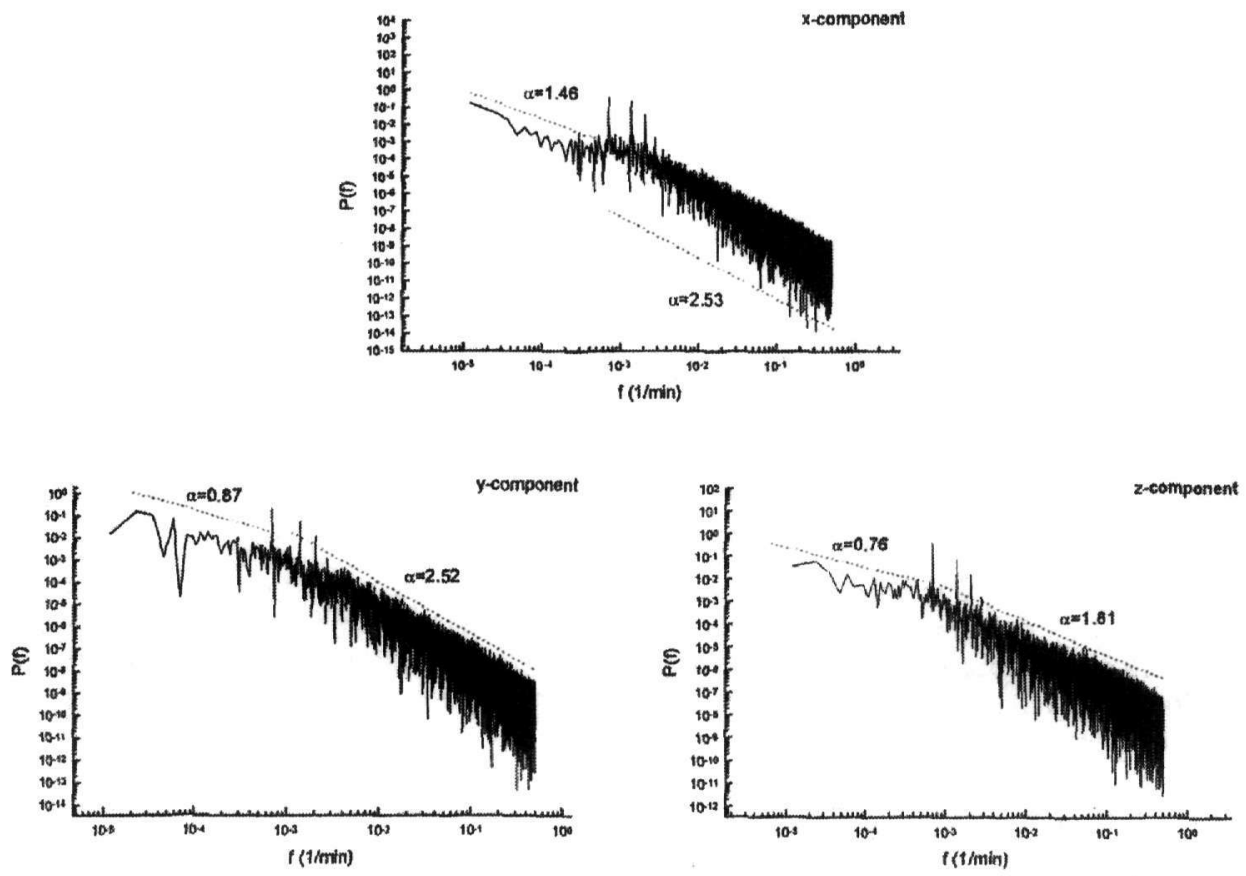

Figure 2. Power spectra of the signals plotted in Fig. 1. 
We performed the Higuchi analysis, which reveals the property of fractal curve in a time series, by means of the estimation of its fractal dimension D (Burlaga and Klein, 1986; Higuchi, 1988). A new time-series is constructed from the given time series $X(i),(i=1,2, \ldots ., N)$,

$$
X_{\tau}^{m} ; X(m), X(m+\tau), X(m+2 \tau), \ldots, X(m+[(N-m) / \tau] \tau) ;(m=1, \ldots \ldots \tau)
$$

where [ ] denotes Gauss' notation. The length of the curve is defined as

$$
L_{m}(t)=\left\{\left(\sum_{i=1}^{[(N-m) / \tau]} \mid X(m+i \tau)-X(\mu+(l-1) \tau \mid) \frac{N-1}{[(N-m) / \tau] \tau}\right\} \frac{1}{\tau}\right.
$$

The average value $\left\langle L(\tau)>\right.$ over $\tau$ sets of $L_{m}(\tau)$ is defined as the length of the curve for the time interval $\tau$. If $\left\langle L(\tau)>\propto \tau^{-D}\right.$, within the range $\tau_{\min } \leq \tau \leq \tau_{\max }$ then the curve is fractal with dimension $D$ in this range.

Fig. 3 shows the results of the Higuchi analysis. As observed in the power spectral densities, we can recognize the presence of two scaling behaviors in each signal, with significantly different estimates for the fractal dimension $D$, which assumes values ranging between 1.1 and 1.2 for the first scaling regime, and values around 1.9 for the second scaling region. Furthermore, the 24-hour oscillation is clearly identifiable, especially at high timescales $\tau$.
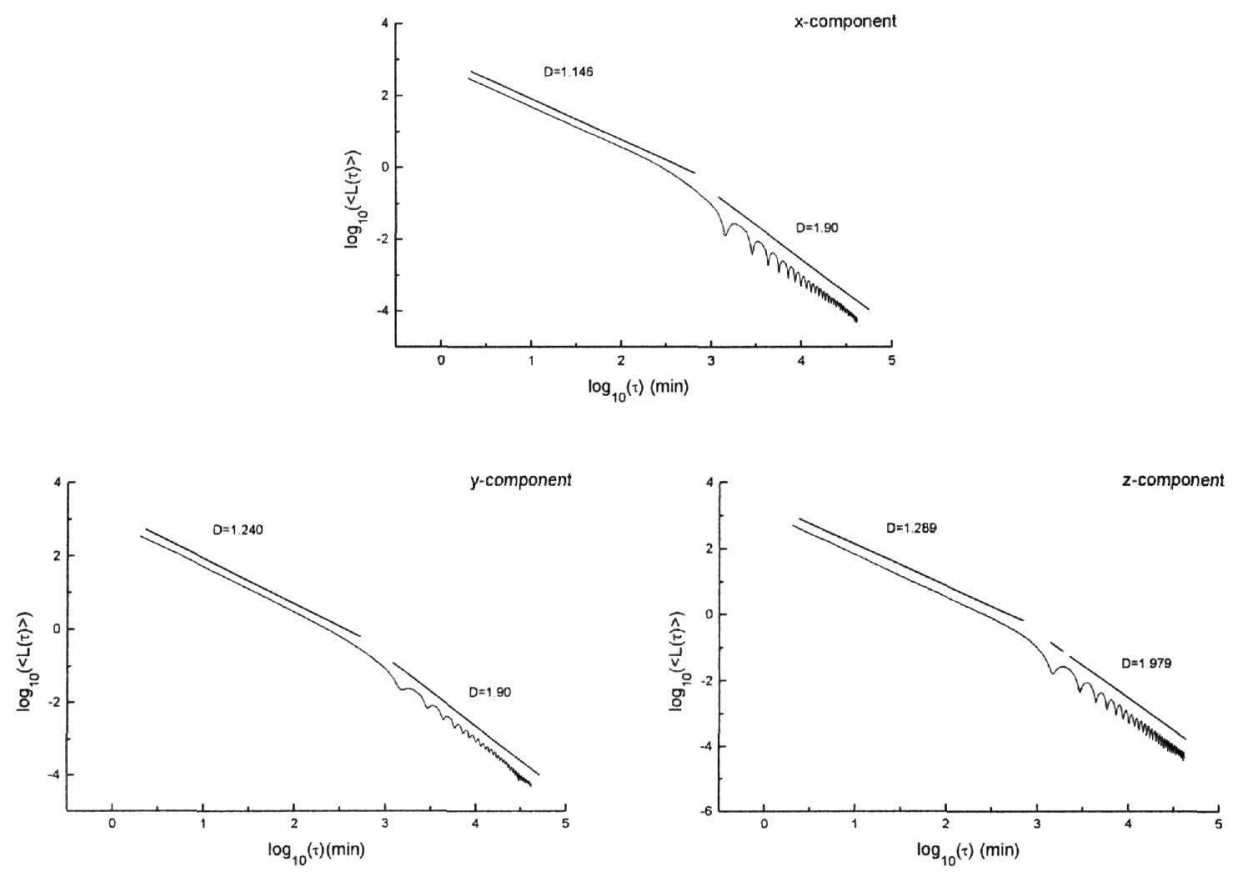

Figure 3. Higuchi analysis of the signals plotted in Fig. 1.

We finally performed the Hurst R/S analysis (Feder, 1988). The Hurst exponent $H$, defined in the following relation, provides information about persistent/antipersistent long-range correlations in a time series.

$\frac{R(m)}{S(m)} \propto m^{H}$ 

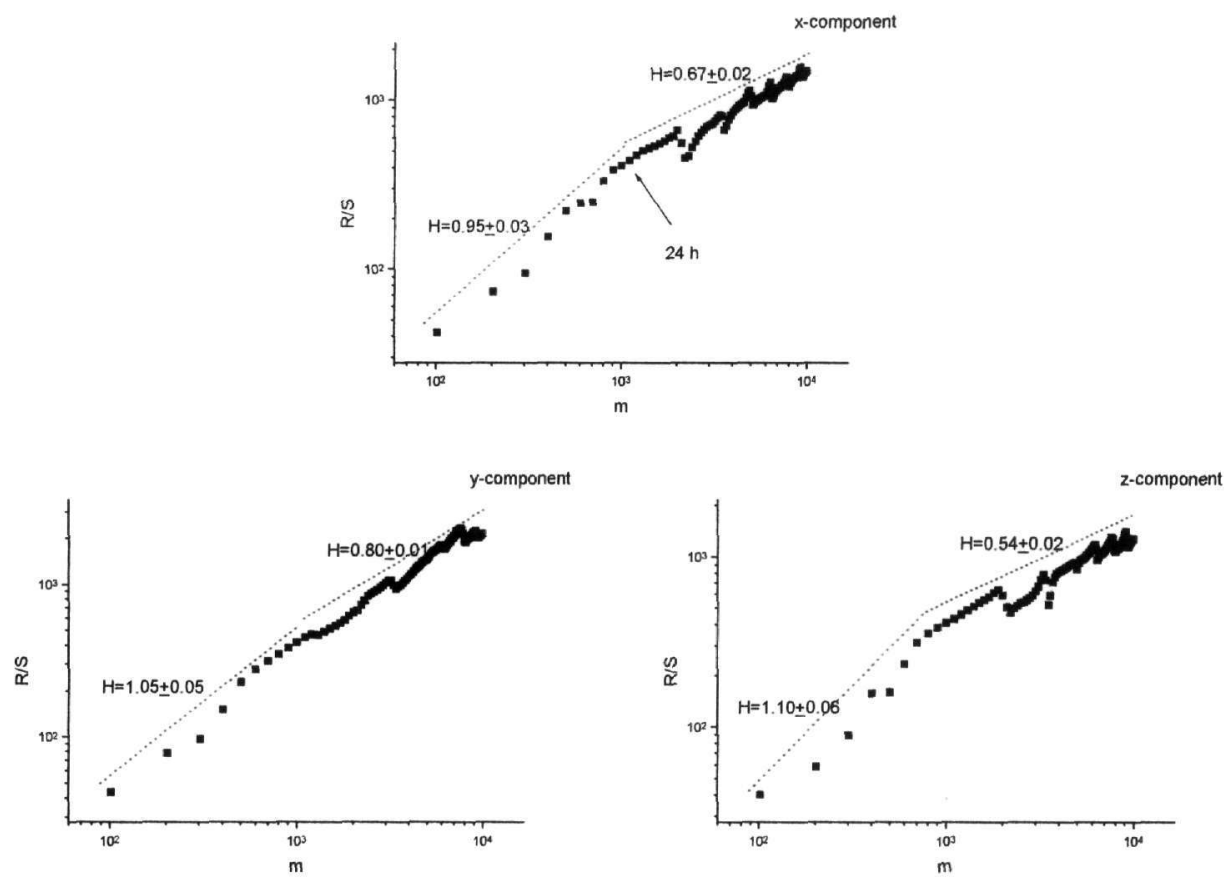

Figure 4. Hurst R/S analysis of the signals plotted in Fig. 1.

The range $R(m)$ is the difference between the minimum and the maximum accumulated departure $X$ and $S(m)$ denotes the standard deviation over the period $m$. For a purely random processes, with no correlations among their increments, $H=0.5$. For other stochastic processes, $H$ can be less or more large than 0.5 , indicating in both cases the presence of correlation. Such a correlation is positive if $\mathrm{H}>1 / 2$, that is increasing (decreasing) fluctuations in the past imply on the average increasing (decreasing) fluctuations in the future. This feature leads to persistence; the signal appears rather smooth and shows seemingly regular patterns. Persistence is useful to describe processes generated by positive feedback mechanisms. Fig. 4 shows the R/S analysis performed on the ULF geomagnetic data of fig. 1. As observed in the previous analyses, the appearance of two scaling regimes is clear, with a crossover located at about 24 hours. Furthermore, the fluctuations of the signals seem persistent at all timescales $\mathrm{m}$, with the short-time scale interactions more persistent than those at long-time scales, as indicated by the value of the Hurst exponent $\mathrm{H}$, that assumes larger values at small time scales. The 24-hour oscillation is also visible at longtimescales, indicating the persistence of such modulation at long time scales.

\subsection{Multifractal analysis}

\subsubsection{Method}

Many authors have developed the multifractality and its formalism, applying it to several fields of the scientific research (Mandelbrot, 1974; Meneveau and Sreenivasan, 1991). Firstly, the so-called partition function $Z(q, \varepsilon)$ has to be defined,

$Z(q, \varepsilon)=\sum_{i=1}^{N_{\text {ooxese }}(\varepsilon)}\left[\mu_{i}(\varepsilon)\right]^{q}$ 
The quantity $\mu_{\mathrm{i}}(\varepsilon)$ is a measure and it depends on $\varepsilon$, the size or scale of the boxes used to cover the sample. The boxes are labeled by the index $i$ and $N_{\text {boxes }}(\varepsilon)$ indicates the number of boxes of size $\varepsilon$ needed to cover the sample. The exponent $q$ is a real parameter that indicates the order of the moment of the measure. The choice of the functional form of the measure $\mu_{i}(\varepsilon)$ is arbitrary, provided that the most restrictive condition $\mu_{i}(\varepsilon) \geq 0$ is satisfied. In the case presented in this paper, we use the following definition of measure:

$$
\mu_{i}(\varepsilon)=\frac{1}{M} \sum_{j=1}^{N_{\text {box }}}|x|_{j}
$$

where $|x|_{j}$ is the $j$-th absolute value of the signal $x$ inside the box i. $M=\sum_{k=1}^{N}|x|_{k}$ is the sum of all the absolute values, and, therefore, it performs as a normalization constant, so that $\sum_{\mathrm{i}} \mu_{\mathrm{i}}(\varepsilon)=1$; with this normalization, the measure $\mu_{i}(\varepsilon)$ can be interpreted as probability.

The parameter q can be considered as a powerful microscope, able to enhance the smallest differences of two very similar maps (Diego et al., 1999). Furthermore, q represents a selective parameter: high values of $q$ enhance boxes with relatively high values for $\mu_{i}(\varepsilon)$; while low values of $q$ favor boxes with relatively low values of $\mu_{\mathrm{i}}(\varepsilon)$. The box size $\varepsilon$ can be considered as a filter, so that big values of the size is equivalent to apply a large scale filter to the map. Changing the size $\varepsilon$, one explores the sample at different scales. Therefore, the partition function $Z(q, \varepsilon)$ furnishes information at different scales and moments.

The generalized dimension are defined by the following equation

$$
D(q)=\lim _{\varepsilon \rightarrow 0} \frac{1}{q-1} \frac{\ln Z(q, \varepsilon)}{\ln \varepsilon}
$$

$D(0)$ is the capacity dimension; $D(1)$ is the information dimension, and $D(2)$ is the correlation dimension. An object is called monofractal if $D(q)$ is constant for all values of $q$, otherwise is called multifractal. In most practical applications the limit in Eq. 6 cannot be calculated, because we do not have information at small scales, or because below a minimum physical length no scaling can exist at all (Diego et al., 1999). Generally, a scaling region is found, where a power-law can be fitted to the partition function, which in that scaling range behaves as

$$
Z(q, \varepsilon) \propto \varepsilon^{\tau(q)}
$$

The slope $\tau(q)$ is related to the generalized dimension by the following equation:

$\tau(q)=(q-1) D(q)$

An usual measure in characterizing multifractals is given by the singularity spectrum or Legendre spectrum $f(\alpha)$, that is defined as follows. If for a certain box $j$ the measure scales as

$$
\mu_{\mathrm{j}}(\varepsilon) \propto \varepsilon^{\alpha_{j}}
$$

the exponent $\alpha$, which depends upon the box j, is called Hölder exponent. If all boxes have the same scaling with the same exponent $\alpha$, the sample is monofractal. The multifractal is given if different boxes scale with different exponents $\alpha$, corresponding to different strength of the measure. Denoting as $S_{\alpha}$ the subset formed by the boxes with the same value of $\alpha$, and indicating as $N_{\alpha}(\varepsilon)$ the cardinality of $S_{\alpha}$, for a multifractal the following relation holds:

$$
N_{\alpha}(\varepsilon) \propto \varepsilon^{-f(\alpha)}
$$

By means of the Legendre transform the quantities $\alpha$ and $f(\alpha)$ can be related with $q$ and $\tau(q)$ : 


$$
\alpha(q)=\frac{d \tau(q)}{d q}
$$

$f(\alpha)=q \alpha(q)-\tau(q)$

The curve $f(\alpha)$ is a single-humped function for a multifractal, while reduces to a point for a monofractal. To be able to make more quantitative statements concerning possible differences in Legendre spectra stemming from different signals, it is possible to fit, by a least square method, the spectra to a quadratic function around the position of their maxima at $\alpha_{0}$ (Shimizu et al., 2002):

$f(\alpha)=A\left(\alpha-\alpha_{0}\right)^{2}+B\left(\alpha-\alpha_{0}\right)+C$

Parameter B measures the asymmetry of the curve, which is zero for symmetric shapes, positive or negative for left-skewed or right-skewed shapes respectively.

Another parameter is the width of the spectrum, that estimates the range of $\alpha$ where $f(\alpha)>0$, obtained extrapolating the fitted curve to zero; thus the width is defined as

$W=\alpha_{\max }-\alpha_{\min }$

where $f\left(\alpha_{\max }\right)=f\left(\alpha_{\min }\right)=0$.

These three parameters serve to describe the complexity of the signal. If $\alpha_{0}$ is low, the signal is correlated and the underlying process "loses fine structure", becoming more regular in appearance (Shimizu et al., 2002). The width $W$ measures the length of the range of fractal exponents in the signal; therefore, the wider the range, the "richer" the signal in structure. The asymmetry parameter $\mathrm{B}$ informs about the dominance of low or high fractal exponents respect to the other. A right-skewed spectrum denotes relatively strongly weighted high fractal exponents, corresponding to fine structures, and low ones (more smooth-looking) for left-skewed spectra. Therefore, high maximum, large width and low asymmetry denote a signal with "strong" multifractality degree.

\subsubsection{Results}

In order to quantify how the presence of powerful oscillations can affect the multifractal character of the signals, we firstly removed from the signals the four periodicities, which were observed in the power spectral densities. We therefore, applied the multifractal formalism to the original signals as well as to the residuals. Fig. 4 shows the multifractal spectra $\alpha-f(\alpha)$ for the three ULF signals, both in the original as well as residual case, obtained considering the time scale range from $10^{3} \mathrm{~min}$ to $4 \cdot 10^{4} \mathrm{~min}$ (more than 1.5 decades). By visual inspection, we can observe that the residual time series display a "stronger" multifractality degree, revealed, in particular, by the wider spectrum. In order to quantify such degree of multifractality, we fitted the multifractal spectra by means of a parabolic function centered on the maximum of each spectrum. The results are shown in Fig. 5: the three residual signals are characterized by higher maximum, larger width and lower asymmetry than those of the original ones. This indicates that the multifractality degree of the signals is strongly reduced by the presence of powerful oscillations. The presence of strong periodicities in the original signals is due to an external origin, correlated with diurnal and semidiurnal processes (mainly, temperature and air pressure); these phenomena act as "regulators" of the dynamics of the ULF signals, which lose their finer time structure becoming less complex.

Furthermore, the vertical component of the ULF residual geomagnetic field is characterized by a stronger multifractality degree than the horizontal component. Maybe, the different character could be put in relation with the higher sensitivity of the horizontal components to low-frequency ionospheric disturbances (Karakelian et al. 2000). 

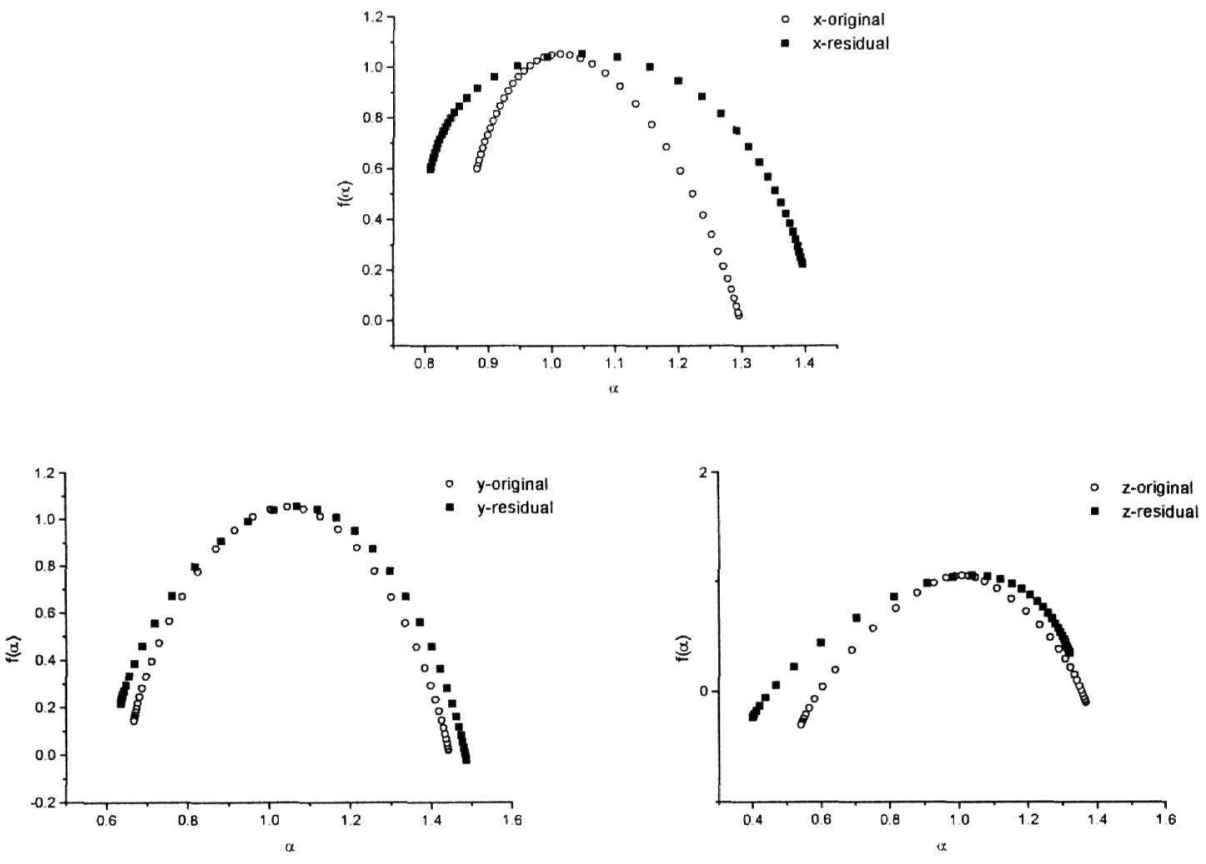

Figure 4. Legendre spectra of the original and residual signals.
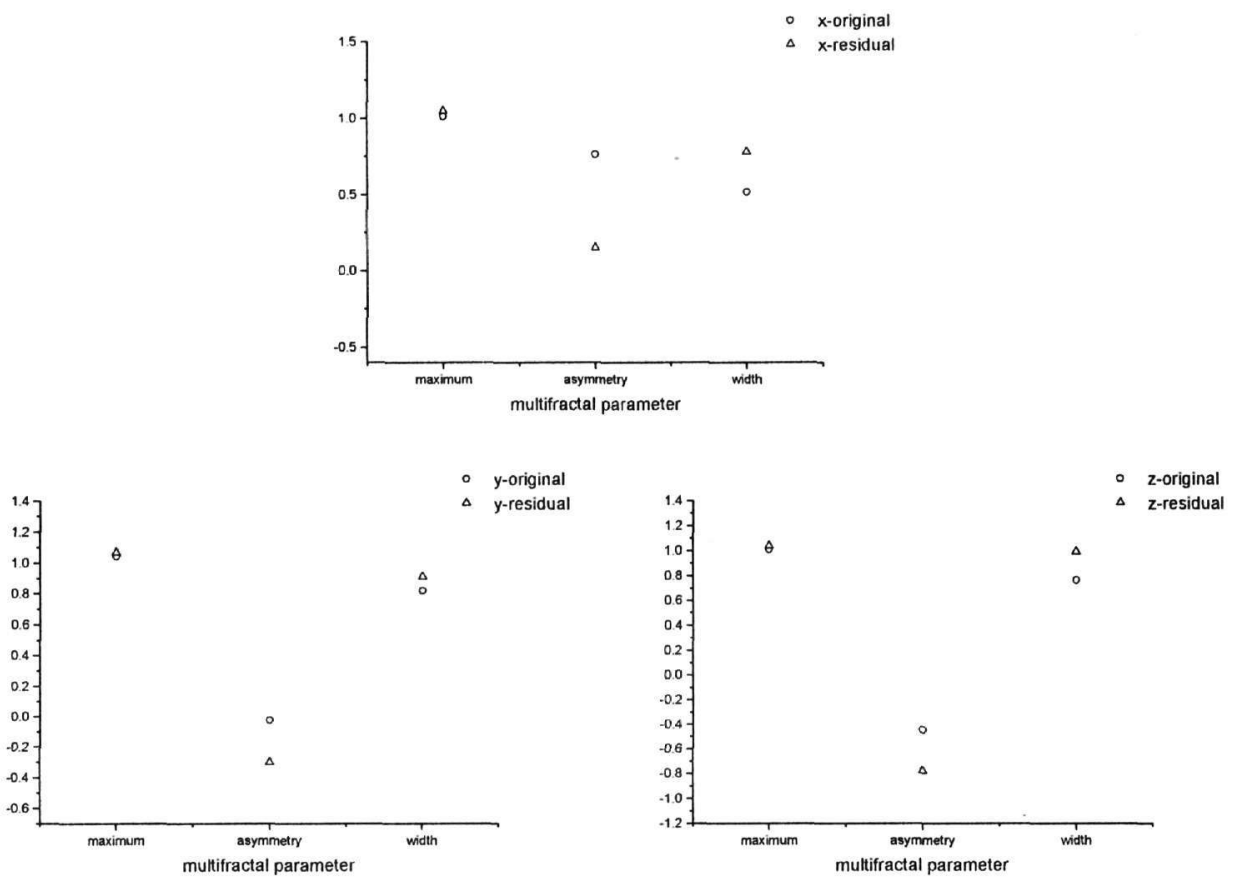

Figure 5. Multifractal parameters of the original and residual signals. 


\section{CONCLUSIONS}

The investigation of the time scale properties of the three-component-ULF geomagnetic field, measured in Crete, Greece, one of the most seismically intense areas of the viorld, has put in evidence very evident fractal features, indicating the presence of $1 / f^{\alpha}$-like long-range correlations. On time scales going from minutes to weeks, the signals, although modulated mainly by diurnal and semidiurnal cycles, show the presence of two main scaling regimes characterized by scaling exponents denoting persistent temporal fluctuations, that indicate that the signals are governed by positive feedback mechanisms. The Legendre spectra have shown a strong multifractality degree for all the signals, from which the diurnal and semidiurnal periodicities have been removed. The vertical component, furthermore, evidences the highest multifractal character.

The multifractal approach could be more advantageous respect to monofractal analysis from extraction of the earthquake precursory signatures. The supporting argument for that is the qualitative analogy between the process of crack propagation and multifractal structure generation, which have been established recently (Kiyashchenko et al., 2003). The multifractal spectrum is characterized by important generalized features of the time series as information entropy and the high-order fractal dimensions, which can be sensitive to earthquake preparation processes. Application of the multifractal approach for study of the evolution of the regional seismicity distribution before a number of the large earthquakes of Japan and Southern California showed specific precursory dynamics of those parameters before the major shocks (Kiyashchenko et al., 2003). So, it is possible to expect the corresponding precursory behavior in the multifractal characteristics of ULF geomagnetic fields in seismo-active regions. The multifractal properties of the ULF signals longer than those investigated in this work suggest us to analyze longer samples of measures in order to assess the variation of the multifractal features of ULF geomagnetic signals. At the same time, we believe that a similar analysis, carried out on data coming from different sites located in the same zone and from sites belonging to different seismic areas might be very interesting to get insight on the link among multifractal properties, features peculiar to the specific site, local tectonics and very general properties of the ULF geomagnetic signals.

\section{REFERENCES}

Baker C., Hatzfeld D., Lyon-Caen H., Papadimitriou E. and Rigo A., 1997. Earthquake mechanism of the Adriatic Western Greece: implications for the oceanic subduction-continental collision transition, Geophys. J. Int. 131, 559-594.

Burlaga L. F., and Klein L. W., 1986. Fractal structure of the interplanetary magnetic field, J. Geophys. Res. 91 , 347-351.

Diego J. M., Martinez-Gonzales E., Sanz J.L., Mollerach S. and Mart V.J., 1999. Partition function based analysis of cosmic microwave background maps. Mon. Not. R. Astron. Soc. 306, 427-436.

Feder J., 1988. Fractals, Plenum Press, New York, p. 181.

Hayakawa M., Ito T. and Smirnova N., 1999. Fractal analysis of ULF geomagnetic data associated with the Guam earthquake on August 8, 1993, Geophys. Res. Lett. 26, 2797-2800.

Hayakawa M., Ito T., Hattori K. and Yumoto K., 2000. ULF electromagnetic precursors for an earthquake at Biak, Indonesia on February 17, 1996, Geophys. Res. Lett. 27, 1531-1534.

Higuchi T., 1988. Approach to an irregular time series on the basis of the fractal theory, Physica D 31, 277-283.

Karakelian D., Klemperer S.L., Fraser-Smith A.C. and Beroza G.C., 2000. A transportable system for monitoring ultra-low frequency electromagnetic signals associated with earthquakes. Seis. Res. Lett. 71, 423-436.

Kiyashchenko D., Smirnova N., Troyan V. and Vallianatos F., 2003. Multifractal analysis of the regional seismicity dynamics before the large earthquakes, precursory evolution of spatial and temporal inhomogeneity of the seismicity distribution. Nat. Hazards Earth Sys. Sci. 3, 285-298.

Kopytenko YuA, Matiashvily TG, Voronov PM, Kopytenko EA and Molchanov OA. 1993. Detection of ULF emission connected with the Spitak earthquake and its aftershock activity based on geomagnetic pulsations data at Dusheti and Varziya observatories. Phys. Earth Planet. Int. 77, 85-95.

Korepanov V., Berkman R., Rakhlin L., Klymovych Ye., Prystai A., Marussenkov A. and Afanassenko M., 2001. Advanced field magnetometers comparative study, Measurement 29, 137-146.

Mandelbrot BB. 1974. Intermittent turbulence in self-similar cascades: divergence of high moments and dimensions of the carrier. J. Fluid Mech 62, 331-358.

Meneveau C and Sreenivasan KR, 1991. The multifractal nature of turbulent energy dissipation. J. Fluid Mech. $224,429-484$. 
Molchanov OA, Kopytenko YuA, Voronov PM, Kopytenko EA, Matiashvili TG, Fraser-Smith AC and Bernardy A., 1992. Results of ULF magnetic field measurements near the epicenters of the Spitak $\left(M_{S}=6.9\right)$ and Loma Prieta $\left(M_{S}=7.1\right)$ earthquakes: Comparative analyses. Geophys. Res. Lett. 19, 1495-1498.

Papazachos B. C., 1990. Seismicity of the Aegean and surrounding area, Tectonophysics 178, 287-308.

Shimizu Y., Thurner S. and Ehrenberger K. 2002. Multifractal spectra as a measure of complexity in human posture. Fractals 10, 103-116.

Stanley HE, Amaral LAN, Goldberger AL, Havlin S, Ivanov PCh and Peng C-K. 1999. Statistical physics and physiology: Monofractal and multifractal approaches. Physica A 270, 309-324.

Tzanis A., Vallianatos F. and Gruszow S., 2000. Identification and discrimination of transient electric earthquake precursors: Fact, fiction and some possibilities, Phys. Earth Planet. Int. 121, 223-248. 